\title{
Predicting Tourism Demands by Google Trends: A Hidden Markov Models Based Study
}

\author{
Uwimana Claude \\ School of Computer and Information Technology, Beijing Jiaotong University, \\ China \\ 17129130@bjtu.edu.cn
}

\begin{abstract}
This article explores the usefulness of information from Google trends to predict the tourism demands. When a tourist interacts with the internet through a search engine, a website, or a social media platform, the traces of the interaction can be captured, stored, and analyzed. Research on most visited cities on the world showed how Amsterdam become a standout among the most visited city on the planet. Related works demonstrated that Google Trends have some values to predict the tourism industry, our new idea for this study is to utilize our model Hidden Markov Model (HMM) with Google Trends data and historical data from the electronic database Central Bureau Statistic (CBS) StatLine 2018 to predict the tourism demands in Amsterdam and to compare our method with existing methods. The search engine user needs to utilize search queries (keywords) identified with the tourism industry in Amsterdam will be extracted using application programming interface(API). We have trained and tested data by the Hidden Markov Model to predict next month tourists number in Amsterdam, For the two existing methods we tune their parameters to get the best results. Our experiments over real data from CBS StatLine demonstrate that our method not only outperforms the traditional and existing methods but also provides controllability to tourism prediction.
\end{abstract}

Keywords: Social media text mining, google trends, tourism prediction, hidden Markov model.

\section{Introduction}

In recent years' internet search engines and social media have become important in the way people inform themselves. When people type in queries on search engines or use search query through social media they implicitly express their interest in the object of the query (Yang et al., 2014; Yang et al., 2015). This research provides a framework for predicting tourism demands using Google Trends. As we know that 
tourism is a growing industry, according to the Organization for Economic Cooperation and Development (OECD) 2018, (Rödel E, 2017) It demonstrates that the tourism industry is now responsible for $9 \%$ of the domestic product and employment worldwide. Additionally, in the Netherlands, the travel industry is developing (Rödel E, 2017).

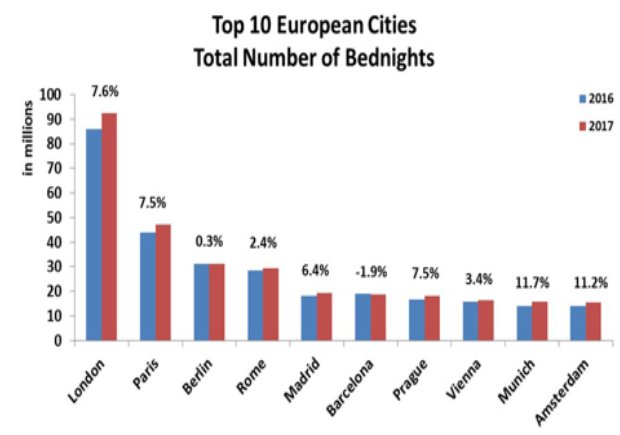

Fig. 1: Top 10 most visited European cities.

Google has developed a tool called Google Trends that helps identify keywords with the most search volume (Volcheck et al., 2018). In particular, search query data provide valuable information about tourists' intention, interests, and opinions. Tourists use search engines and social media to obtain weather and traffic information and to plan their routes by searching for hotels, attractions, travel guides, and other tourists' opinions. We use search query because when a tourist interacts with the Internet through a search engine, a website, or a social media platform, the traces of the interaction can be captured, stored, and analyzed (Paper, 2016).

This study aims to investigate the opportunity to predict the number of visits based on online tourist search. What people write on their microblogs reflects their intent and desire relating to most of their common day interests (Length, 2016). Taking this as strong evidence, we hypothesize that search queries of the person can also be treated as a source of strong indicator signals for predicting their places of visits, predict related with the three info highlights which are the date, tourists number and locations those are from Google Trends dataset, the yield the mark is number of Tourists in Amsterdam. From May 29th, 2016 up 31st December,2018 aggregate of 265,307 searchers from five nations (United Kingdom, Germany, France, Belgium, Sweden), utilized with six predictive power search queries (Amsterdam, Hotel Amsterdam, visit Amsterdam, travel Amsterdam, city trip Amsterdam, Holiday Amsterdam) have been trained and tested by Hidden Markov Model. The advantage of utilizing inquiry patterns to predict tourist data is two-fold. First, query trends can utilize data up to the day before the forecast computation, which could possibly be very significant in this setting because of the slacks in the 
distribution of the official measurement.

\subsection{Tourist Journey}

Before the tourist journey for the tourism sector is defined, it is important to understand the general definition of tourist behavior. The process by which a tourist chooses the place to visit or interests and opinions is defined as the tourist behavior process (Song and Li, 2008; Dijkman and Ipeirotis, 2015; Nenonen et al., 2008).

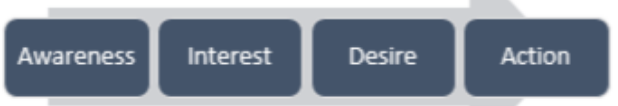

Fig. 2: Customer journey

The city of Amsterdam publishes reports concerning the tourism industry branch altogether on a yearly base. The reports concern trends in the tourism industry, incoming tourism industry, Dutch tourism industry, the impact of the tourism industry on the Dutch economy, employment opportunities in the branch and diversion. In these reports, the city presents an information sheet about the previous year and predicts the next year according to information from last year (Rödel, 2017).

The reports give no understanding of the particular determining methods used to structure these predictions ( $\mathrm{Li}$ et al., 2017). However, there are some challenges regarding the analysis, capture, search, sharing, storage, transfer, and visualization and information privacy of big data, these challenges require new programs or technologies to uncover hidden values (SMITH, 1946), Google Trends might be one of those programs needed to enlarge the advantage of using big data to predict tourism demand.

This has prompted the following research question for this study:

1. What extent is the data provided by Google Trends useful for predicting night passes in hotels in Amsterdam?

To be able to answer this central research question the following sub-questions will be answered:

A. What predicting methods are utilized for predicting the travel industry demands now?

B. What is Google Trends and how does it work?

C. Is Google Trends better predictor than the traditional forecasting method for tourism in Amsterdam?

\section{Literature review}

This section reviews relevant studies in the areas of tourism demand and 
socioeconomic activity forecasting with search engine query data (Claveria and Torra, 2014). Specifically, a few recent studies on forecasting tourism demand with search engine data are also discussed. A decision making process in the travel industry involves three following stages: information search, travel planning, and trip arrangement of action (Cuhadar, 2014; Fesenmaier et al., 2016). The primary goals of information search activities are to increase the overall quality of a future trip and to decrease a perceived high risk, related to future travel.

The development of ICTs and mobile computing devices have made the Internet the main source of travel information. Search engines, such as Google and Baidu, are the primary sources of tourism information, which shape tourist perceptions on attraction image and encourage travel decisions.

\subsection{Econometric models}

Econometric models are models that attempt to replicate the important structures of the real world (Song, 2010; Wijnhoven and Plant, 2017). These models can include any number of simultaneous multiple regression analysis with several interdependent variables. The major advantage of the econometric approach is the ability to analyze causal relations. Basic utilized econometric models in the literature are time-varying parameter (TVP) models, the vector autoregressive (VAR) model and the Error correction model (ECM). About forecasting performances; these models generally predict well although there remains more research to be done to create significant improvements (Khadivi, 2016).

\subsection{Artificial Neural Networks (ANN)}

Artificial neural networks are forecasting methods that are based on simple mathematical models of the brain (Wanjawa and Muchemi, 2014; Time, 2017; Jun et al., 2018; Huang et al., 2005). A neural system comprises an input layer, an output layer, and normally one or more hidden layers. Every one of these layers contains nodes, and these nodes are connected to nodes at neighboring layer(s) (Bursali, 1973). In Artificial neural networks, lagged values of the time series are utilized as contributions to a neural network (like a direct autoregressive model). We think about a feed-forward network with one hidden layer. The forecasts are acquired by a linear combination of the information sources (Nath, 2005).

\section{Methodology}

This study aims for predicting the tourism industry demands, in the form of the touristic night passes in hotels, with Google Trends data and CBS StatLine data with the utilization of Hidden Markov Model HMM. The study takes the form of time-series modeling since it depends on trends and patterns recognized by Google trends. For that this advanced methodology will be utilized, in the first place, the tourist journey theory is utilized to subtract search query terms. It will be researched 
how strong the relationship is between these terms and the pattern of specific tourist's statistics of the city of Amsterdam. Based on this, we attempt to discover different search queries (keywords) for this field of interest. With this structure, the distinction among clarifying and predicting will be made which leads us to the following method questions:

1. What are important keywords for predicting tourists in Amsterdam as indicated by tourist journey theory?

2. What are the important keywords for predicting tourists in a hotel in Amsterdam according to Google Correlate?

\subsection{The Workflow of our Method}

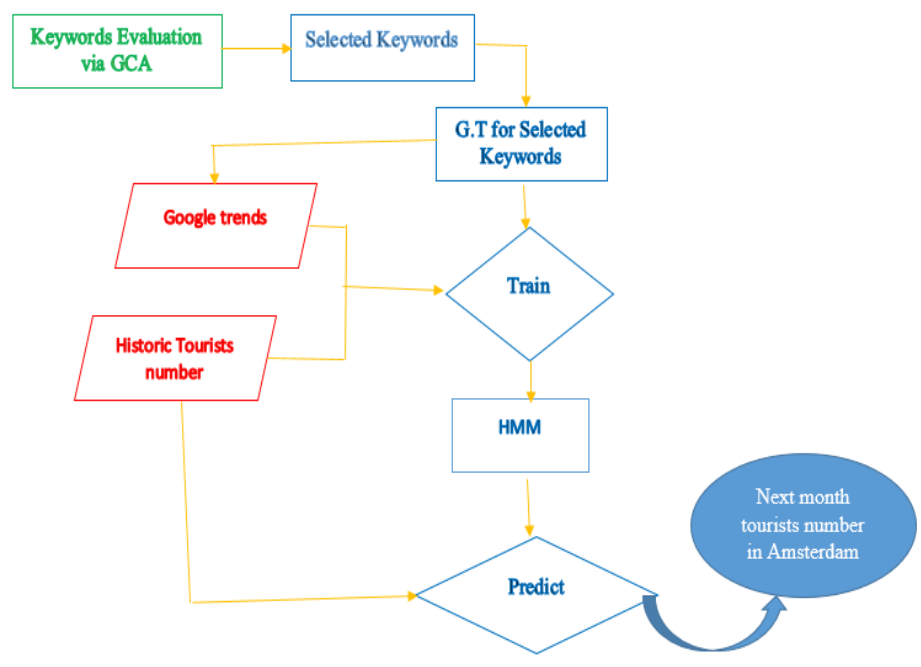

Fig. 3: The workflow of our method.

This mechanism enables the users to interface the forecast procedure, which is required feature from the training view. We propose to utilize Google Trends data with HMM so that we can accomplish high prediction execution as well as acquire great controllability to the tourism industry forecast. Concretely, we first set list of keywords composed with 'Hotel Amsterdam', Flight Amsterdam' 'Train to Amsterdam', 'City trip Amsterdam', Holiday Amsterdam', 'Visit Amsterdam' Tourist info Amsterdam and ' Amsterdam '. Second, we perform Granger Causality Analysis (GCA) between tourism industry index and search query of Google Trends to effectively figure out which search query has the most predictive power on the index. Extensive experiments over real datasets demonstrate that our method not only performs the traditional method, yet in addition gives controllability to the tourism industry forecast. 


\subsection{Data Collection}

Data will be collected from Google Trends and the electronic database CBS StatLine (2018). This study will focus on the calendar years 2016, 2017 and 2018. The data set contains tourist's arrival information for the Netherlands destination are on a monthly level for a total period of thirty-six (36) months. As pointed out in the hypothesis of this study, the search engine user needs to use keywords related to the travel industry, get search query information and select fitting information.

Table.1: Selected keywords and search engine users.

\begin{tabular}{|c|c|c|c|c|c|c|c|}
\hline \multirow{2}{*}{$\begin{array}{c}\text { PERIOD } \\
2016-2018\end{array}$} & \multirow{2}{*}{ COUNTRY } & \multicolumn{5}{|c|}{ KEYWORDS } & \multirow[b]{2}{*}{$\begin{array}{l}\text { Holiday } \\
\text { Am }\end{array}$} \\
\hline & & Amsterdam & $\begin{array}{l}\text { Hotel } \\
\text { Am }\end{array}$ & $\begin{array}{l}\text { Visit } \\
\mathrm{Am}\end{array}$ & $\begin{array}{l}\text { Trave } \\
1 \mathrm{Am}\end{array}$ & \begin{tabular}{|l|} 
City \\
trip Am
\end{tabular} & \\
\hline & Germany & 7506 & 5481 & 6910 & 7361 & 5569 & 7209 \\
\hline & UK & 13152 & 11254 & 10636 & 12452 & 10496 & 13110 \\
\hline & France & 9174 & 7296 & 5680 & 8910 & 5479 & 9006 \\
\hline & Belgium & 12788 & 10264 & 12870 & 12558 & 10215 & 12800 \\
\hline & Sweden & 10981 & 6587 & 4520 & 10941 & 4620 & 10842 \\
\hline $\begin{array}{l}\text { Researcher } \\
\text { Number }\end{array}$ & 265307 & 53601 & 40882 & 29256 & 52222 & 36379 & 52967 \\
\hline
\end{tabular}

The total number of search engine user for all keywords is 265,307 , the keyword has most search records is Amsterdam, have 53601 searches.

\subsection{Hidden Markov Model}

Hidden Markov Model is a predictable state mechanism which has some fixed number of states. It provides a probabilistic framework for modeling a time series of multivariate observations (Mathew et al., 2012; Daniel and Martin, 2018; Pai et al., 2018)

The advantages of HMM can be summarized as:

1. HMM has a strong statistical foundation.

2. It is able to handle new data robustly, computationally efficient to develop and evaluate.

For whatever remains of this research the following notations will be used with respect to HMM:

$\mathrm{N}=$ number of states in the model.

$\mathrm{M}=$ number of distinct observation symbols per state.

$\mathrm{T}=$ length of observation sequence.

$\mathrm{O}=$ observation sequence $O_{1}, O_{2}, O_{3} \ldots O_{T}$

$\mathrm{A}=\left\{a_{i j}\right\}$ transition matrix, where $\mathrm{a}_{\mathrm{ij}}$ represents to the progress probability from state $i$ to state $j$.

$\mathrm{B}=\left\{b_{i}\left(O_{t}\right)\right\}$ Observation emission matrix, where $b_{i}\left(O_{t}\right)$ represent to the 
probability of observing $j \cdot \Pi=(A, B, \pi)$ the prior probability, where $\pi_{i}$ represent to the probability of being in state $i$ toward the start of the experiment, at time $\mathrm{t}=1$.

$\mathrm{A}=(A, B, \pi)$ the overall HMM model. As mentioned above the HMM is described by N, M, A, B and $\pi$ the $\mathrm{a}_{\mathrm{ij}} ; b_{j}\left(O_{t}\right)$ and $\pi_{i}$ have the properties.

$$
\sum_{j} \mathbf{a}_{\mathrm{ij}=\mathbf{1}} \boldsymbol{x} \boldsymbol{f} \sum_{i} \pi_{i}=1 \text { and } \mathbf{a}_{\mathrm{ij}} b_{i}\left(O_{t}\right) \boldsymbol{\pi}_{\boldsymbol{i}} \geq \mathbf{0} \text { for all } \boldsymbol{i}, \boldsymbol{j}, t
$$

\subsection{Artificial Neural Network (ANN)}

ANN is a mathematical model or computational model based on biological neural networks. It consists of an interconnected group of artificial neurons and processes information using a connectionist approach to computation. He most important neural network type is the Multi-Layer Perceptron (MLP) with the strict feedforward architecture of three layers. The connections between inputs and outputs are typically made via one or more hidden layers of neurons or nodes. The input layer is defined to assume the values of the input vector and does not perform any additional computation, the hidden layer of neurons or nodes is fully connected to the input and output layers and usually uses the sigmoid function as an output function. Figure 3.3 shows a typical ANN with three inputs, and one hidden layer of two neurons and one output (Polykalas et al., 2013).
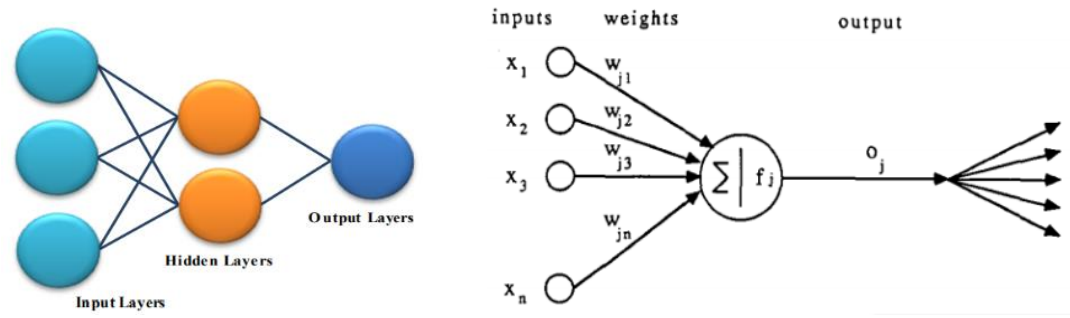

Fig. 4: Typical ANN with Three Inputs, One Hidden Layer of Two Neurons.

The "generalized delta rule" is suggested by Rumelhart et al. and gives a recipe for adjusting the weights on the internal units based on the error at the output. To be more specific, let

$$
\mathrm{E}_{\mathrm{P}}=\frac{1}{2} \sum_{\mathrm{J}}\left(\mathrm{t}_{\mathrm{pj}}-\mathrm{O}_{\mathrm{pj}}\right)^{2}
$$

Be the measure of the error in pattern $\mathrm{p}$ and let $E=\sum p E p$ be the overall measure of the Error, where $t p j$ is the target output for the component of the actual output pattern produced by the network representation with input pattern $\mathrm{p}$. The network is specified as

$$
\underset{\mathrm{pj}}{\mathrm{o}}=\underset{\mathrm{j}}{\mathrm{f}}\left(\text { net }_{\mathrm{pj}}\right)
$$




\subsection{Vector Auto-Regressive (VAR)}

The vector auto regression (VAR) model is one of the most successful, flexible, and easy to use models for the analysis of multivariate time series. The VAR model is useful for describing the dynamic behavior of financial time series and for forecasting. There are many studies about modeling financial time series with VAR models [7].

\subsection{Hidden Markov Model as a solution}

In this section we develop an HMM-based tool for time series forecasting, for instance, to predict tourism demands, while implementing the HMM, the choice of the model, choice of the number of states and observation symbol (continuous or discrete or multi-mixture) become a hard task.

Our observation here being constant as opposed to discrete, we choose experimentally upwards of 3 mixtures for each state for the model destiny. For the prior probability $\pi_{i}$, a random number was chosen and normalized so that $\sum_{i=1}^{N} \pi_{i}=1$.

The dataset being continuous, the probability of emitting symbols from a state cannot be calculated. Therefore, a three-dimensional Gaussian distribution was initially chosen as the observation probability destiny function. Thus we have

$$
\mathrm{b}_{\mathrm{j}}\left(\mathrm{o}_{\mathrm{j}}\right)=\sum \mathrm{c}_{\mathrm{jmn}}\left[\mathrm{o}, \mu_{\mathrm{jm}} \mathrm{u}_{\mathrm{jm}}\right] 1 \leq \mathrm{j} \leq \mathrm{N}
$$

$o=$ vector of observations being demonstrated.

$c_{i m}=$ mixture coeff. for the $\mathrm{m}$-th mixture in state $\mathrm{j}$, where $\sum_{m_{-1}}^{M} c_{i m=1}$

$\mu_{i m}=$ mean vector for the $\mathrm{m}$-th blend segment in state $\mathrm{j}$.

$\aleph=$ Gaussian density.

For training the model, three years $(2016,2017,2018)$ Google Trends data and information from CBS StatLine were utilized and recent last one year's data were utilized to test the efficiency of the model.

\subsubsection{Keywords Extraction and Evaluation}

In this section, we extract and evaluate Google Trends keyword. Our aim is predicting tourist number in Amsterdam, so we focus on analyzing the keywords in general. First, we evaluate the predictive power of different keywords via GCA and determine the most predictive Keywords. Because of the massive amount of keyword records, we used a CPA to reduce them. The filter method removes keywords containing contain zero records and keeps keywords containing records.

Formally, the following two equations hold:

$$
\begin{gathered}
\mathrm{y}_{t}=y o+\sum_{i=1}^{l a g} y_{i} Y_{t-i}+\varepsilon_{t} \\
\mathrm{y}_{t}=y o+\sum_{i=1}^{l a g} y_{i} Y_{t-i}+\sum_{i=1}^{l a g} x_{i} X_{t-i}+\varepsilon_{t}
\end{gathered}
$$


Above, and are time variables (month), and lag is the upper bound of lagged months. We perform GCA the growth of the tourism industry volume record (Y) and keyword $(\mathrm{X})$. We determine the keyword that has the most predictive power and its corresponding lagged value as according to the following two rules:

1. Find which is at a statistically significant level ( $p$ value $\leq 0.1$ ).

2 . Find which reduces significantly comparing to its precursor (distinction $\leq 0.25$ ). Definition 1: Given an observation sequence $\mathrm{O}=\{\mathrm{o} 1, \mathrm{o} 2, \ldots, \mathrm{oT}\}$ (showing historical tourists from CBS StatLine), a relative label sequence $L=\{11,12, \ldots, 1 \mathrm{~T}\}$ (demonstrating next month tourists number) and an HMM $\lambda$, the state label pi denotes the most probable label that state $\mathrm{q}_{\mathrm{i}}$ should have. Formally, above, $y_{t i}=p\left(s_{t}=q_{i} \backslash o, \lambda\right)$ means the probability that the HMM $\lambda$ stays at state $\mathrm{q}_{\mathrm{i}}$ at the time, which can be processed by the forward-backward technique.

Definition 2: Given an observation sequence $\mathrm{O}=\{\mathrm{o} 1, \mathrm{o} 2, \ldots, \mathrm{oT}\}$ and an $\mathrm{HMM} \lambda$, the experimental visit rate indicates the part of the time that the HMM $\lambda$ spends at state $q_{i}$ i.e.

$$
v_{i}=\frac{1}{T} \sum_{t=1}^{T} y_{t i}
$$

Definition 3: Given an observation sequence $\mathrm{O}=\{\mathrm{o} 1, \mathrm{o} 2, \ldots, \mathrm{oT}\}$, a relative label sequence, $\mathrm{L}=\{11,12, \ldots, \mathrm{IT}\}$ and an $\operatorname{HMM} \lambda$, the exact state risk ri indicates the rate of erroneous visits to state $\mathrm{q}_{\mathrm{i}}$ Formally,

$$
r_{i}=\frac{\frac{1}{T} \sum_{t=1, l_{t} \neq p i}^{T} y_{t i}}{v_{i}}
$$

\subsubsection{Hidden Markov Model Training}

Given a coverage bound $C_{B}$, numerous observation sequences $Q_{K}$ and a label sequence $L$, we train an HMM and recursively refine the heavy state $q_{h}$ until there is no heavy state remaining. The training process comprises of the following progress:

1. Initialize the root HMM $\lambda 0$ with random parameters, and train it with the set $Q_{K}$ of historical tourists from CBS StatLine and Google trends information. The training algorithm is Baum-Welch variation adjusted observation sequences.

2. Compute state label $\mathrm{p}_{\mathrm{i}}$, empirical visit rate $\mathrm{v}_{\mathrm{i}}$ and empirical state risk $C_{B}$ for each state qi in the root HMM $\lambda 0$. Under the coverage bound $C_{B}$, compute the reject subset RS of the root HMM $\lambda 0$ to identify which state is the heavy state $\mathrm{q}_{\mathrm{h}}$. If there is no heavy state, then the training is done.

3. Initialize a random HMM $\lambda$ random to replace the heavy state $\mathrm{q}_{\mathrm{h}}$, so a refined HMM $\lambda$ refine is obtained. Train the refined HMM $\lambda$ refine with the previous set $Q_{K}$ of multiple observation sequences until it converges. 


$$
\begin{gathered}
\pi_{\mathrm{j}}=\frac{\sum_{\mathrm{i}=1}^{\mathrm{K}} \frac{1}{\mathrm{p}_{\mathrm{i}}}\left(\mathrm{y}_{\mathrm{l}_{\mathrm{j}}}^{(\mathrm{i})}+\sum_{\mathrm{t}=1}^{\mathrm{T}_{\mathrm{i}-1}} \sum_{\mathrm{k}=1, \mathrm{k} \neq \mathrm{h}}^{\mathrm{N}} \zeta_{\mathrm{t}, \mathrm{k}, \mathrm{j}}{ }^{(\mathrm{i})}\right)}{\mathrm{Z}} \\
a_{j k}=\frac{\sum_{i=1}^{K} \frac{1}{p_{i}} \sum_{t=1}^{T_{i-1}} \zeta_{t, k, j}{ }^{(i)}}{\sum_{l=N+1}^{N+n} \sum_{i=1}^{k} \frac{1}{P_{i}} \sum_{t=1}^{T_{i-1}} \zeta_{t, k, l}^{(i)}} \\
b_{j m}=\frac{1}{\sum_{i=1}^{k} \frac{1}{p_{i}} \sum_{t=1, o_{t}^{(i)}=u m}^{T_{i}} y_{t j}^{(i)}} \\
\sum_{i=1}^{k} \frac{1}{p_{i}} \sum_{t=1}^{T_{i}} y_{t j}^{(i)}
\end{gathered}
$$

Above, $\mathrm{Z}$ is the standardization factor of $\pi_{\mathrm{j}}$, and $\left.\left(\xi_{\mathrm{t}, \mathrm{j}, \mathrm{k}}=\mathrm{Ps}_{\mathrm{t}}=\mathrm{q}_{\mathrm{j}}, \mathrm{s}_{\mathrm{t}}+1=\mathrm{q}_{\mathrm{k}} \mid \mathrm{O}, \lambda\right)\right)$ is the probability of HMM $\lambda$ from state $\mathrm{q}_{\mathrm{j}}$ to state $\mathrm{q}_{\mathrm{k}}$ time, which can be efficiently computed by the forward-backward procedure.

4. Compute exact visit rate $v_{i}$ and experimental state risk $r_{i}$ for each state qi in the refined HMM $\lambda$ refine. Under the coverage bound $C_{B}$, compute the reject RS subset of the refined HM $\lambda$ refine to recognize which state is the overwhelming state $\mathrm{q}_{\mathrm{h}}$. If there is a heavy state, go to Step 3 .

\subsubsection{Hidden Markov Model Prediction}

Given a trained stream HMM and another observation sequence $\mathrm{O}=\{01,02, \ldots, \mathrm{oT}\}$, e forecast the last label $\mathrm{l}_{\mathrm{T}}$ in the relative label sequence $\mathrm{L}$ as indicated by $\mathrm{O}$ through recursively finding the most 1 probable state $q_{\text {most }} t$. The prediction procedure comprises the following steps:

1. Find the most probable state $\mathrm{q}_{\text {most }}$ at the last time $\mathrm{T}$ by the computing $\gamma \mathrm{T}_{\mathrm{i}}$ of all states in the root HMM $\lambda 0$.

2. If $\mathrm{q}_{\text {most }}$ is a refined state $\mathrm{q}_{\text {refined }}$, reset the most probable state $\mathrm{q}_{\text {most }}$ by the computing $\gamma \mathrm{T}_{\mathrm{i}}$ of new states added to the following dimension refined HMM $\lambda$ refine, and go to Stage 2.

3. If $\mathrm{q}_{\text {most }}$ is in the reject subset $\mathrm{RS}$, no forecast is made; something else, the label $p_{\text {most }}$ of the state $q_{\text {most }}$ is yield as the prediction result of the last label $p_{\text {most }}$ in the relative according to $\mathrm{O}$.

\section{Conclusion and Proposals}

In this section, we present experimental evaluation results. First, we introduce an experimental datasets and computing environment. Then, we present and analyze the results of the GCA. Finally, we compare our method and two existing methods to demonstrate the advantage of our method. 


\subsection{Statistics of Extracted Keywords}

We extract keywords from Google Trends and keep the extracted more data using different keywords in monthly periods as long have not enough data. Then we get six predictive power keywords. The results of those keywords plotted here.

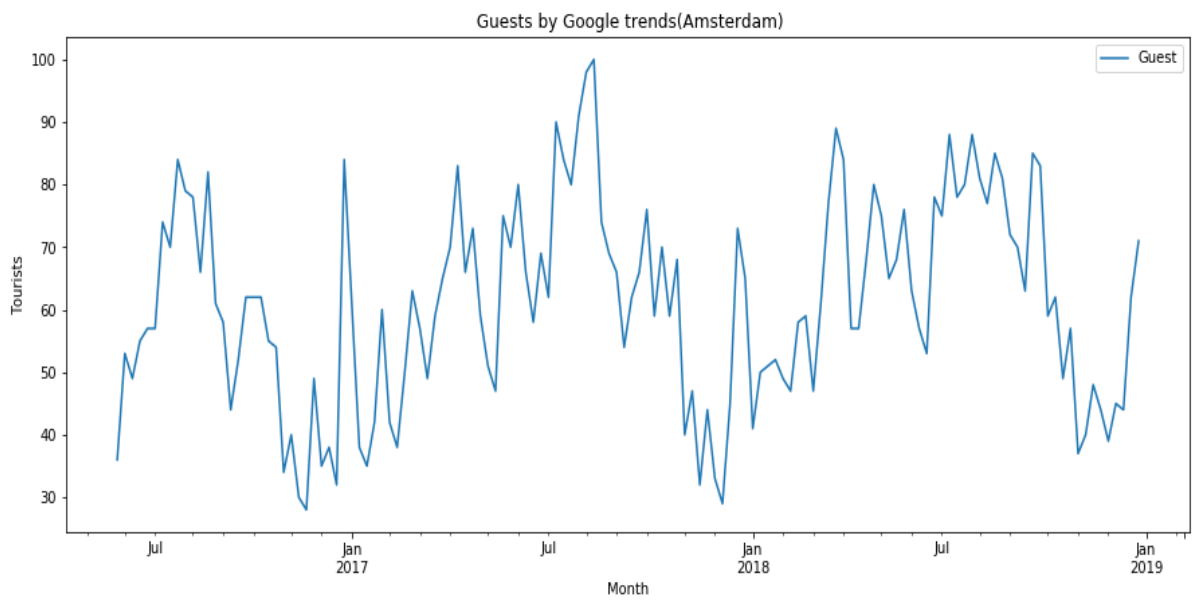

Fig. 5: Keyword “Amsterdam”.

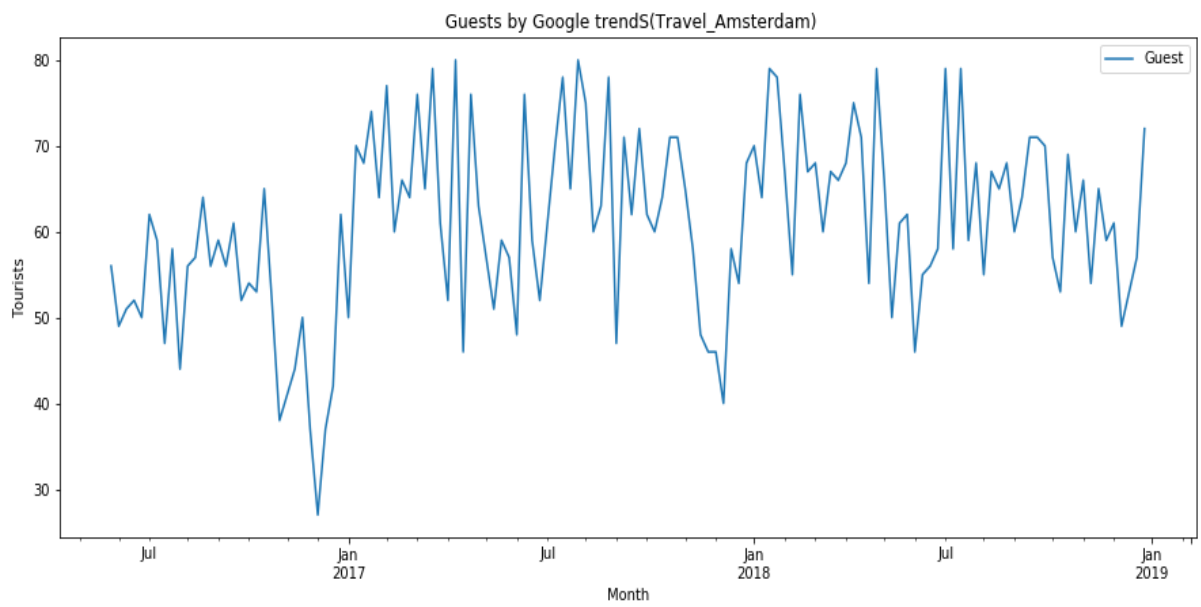

Fig. 6: Keyword "Visit Amsterdam"

\subsection{Results of Granger Causality Analysis}

After effects of Granger Causality Examination, we perform GCA on the extracted and analyze Google Trends data. We treat the Google Trends data volume as time series respectively, while taking every keyword as time series. The range is set from 1 to 3.We present the pvalue results of Google Trends information in Table 4.1.By checking the outcomes in the table, we select three months (i.e., lag=3 ) 
lagged Amsterdam(Am). We use Google Trends keywords as the predictive pointer of the travel industry requests because of following two reasons:

1. Most under three months of lagged Amst. Google Trends keywords achieve a significant level $(\leq 0.1)$, and its value is 0.061 .

2. All pvalue under three months of lagged Amst. Google Trends search query (keywords) decrease significantly (difference 0.25) comparing to pvalue under two months lagged Amst, Google trends keyword.

Table 2: pvalue Results of Google Trend keywords (allpvalue* $\leq 0.1$ )

\begin{tabular}{|c|c|c|c|c|c|c|}
\hline Lag & \multicolumn{5}{|c|}{ Google Trends } \\
\hline Keywords & Amst & Hotel Am & Visit Am & Travel Am & City trip Am & Holiday Am \\
\hline 1 & 0.407 & 0.266 & 0.651 & 0.686 & 0.525 & 0.250 \\
\hline 2 & 0.467 & 0.436 & 0.638 & 0.548 & 0.702 & 0.315 \\
\hline 3 & 0.061 & 0.150 & 0.656 & 0.276 & 0.731 & 0.328 \\
\hline
\end{tabular}

\subsection{Prediction Performance Comparison}

We compare our method and two existing methods, in which two methods exploit Google trends. These two methods and our method all incorporate 3months lagged Am. Our method performs well on Google trends to predict the tourist industry demands. The two methods are:

1. The Vector Autoregressive (VAR) framework treats tourism information and Google Trends as a coordinated vector to make prediction based linear regression.

2. Artificial Neural Network (ANN) utilizes two nodes of "big" and "small" as yield layer and puts the two tourist's information and Google Trends to include a layer to make the prediction. Well as our method is the best model for predicting the tourism industry demands utilizing search engines with controllability.

Table 3: Comparison with two methods using Google Trends.

\begin{tabular}{|c|c|}
\hline MODEL & ERROR RATE(\%) \\
\hline V A R & 28.132 \\
\hline A N N & 33.667 \\
\hline HMM & 24.109 \\
\hline
\end{tabular}

Correlation with existing strategies utilizing Google patterns, as HMM's performance is flexible by the coverage bound $\mathrm{CB}$, we set 3 values between 1.0 and 0.1 for $\mathrm{CB}$ to evaluate HMM. A smaller $\mathrm{CB}$ value means that HMM puts more restriction on prediction output, which prompts a smaller error rate. For the two existing methods, we tune their parameters to get the best results.

All experimental results are presented in Table.3; we can see that among the two existing methods, VAR has the smallest error rates $28.132 \%$. For the three cases, 
by $\mathrm{CB}$ 's an incentive respectively, HMM can get smaller error rates $24.109 \%$ than the two existing methods. Also, with $\mathrm{CB}=0.1 \mathrm{HMM}$ achieves the lowest error rate on all two of datasets. All the more significantly, the error rate of HMM is controllable, while the existing methods do not have such a feature. The results of predicting tourism demands in Amsterdam using Google Trends is plotted here.

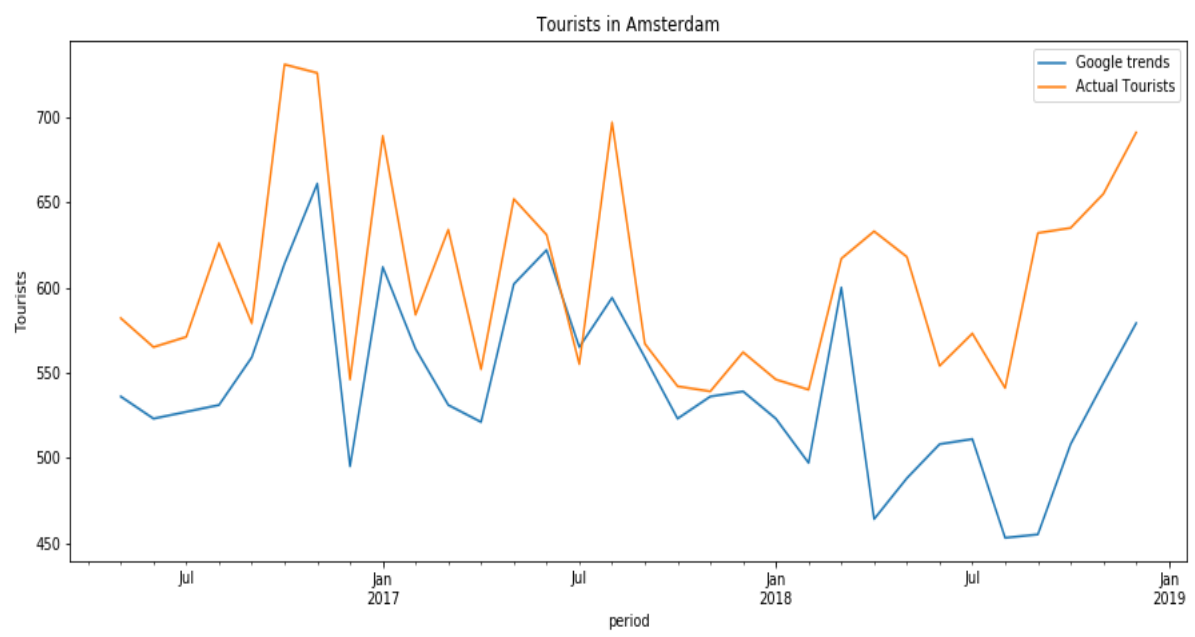

Fig. 7: Result of Predict Tourism demands in Amsterdam.

\section{Conclusions}

We proposed to use Google Trends forecast based on HMM. First, we used six predictive power keywords, at that point we performed GCA between over massive keywords to figure out which Google Trends keywords has the most predictive power on tourism forecast. Finally, we extended method with three months lagged Amsterdam (Amst) Google Trends data not only performs better than existing methods, yet additionally gives a controllability mechanism to the tourist industry prediction.

\section{Acknowledgments}

This research is part of my master's thesis. I thank my master tutor prof. Jitao sang and I thank for Dalin Zhang for his warm help.

\section{References}

Bursali S (1973). Determination of the Minimum Number of Samples for the Compaction Control of Impervious Fills. Tour. Manag. 70 1-15 
Claveria O and Torra S (2014). Forecasting tourism demand to Catalonia: Neural networks vs. time series models Econ. Model. 36 220-8

Cuhadar M (2014). Modelling and Forecasting Inbound Tourism Demand to Istanbul: A Comparative Analysis Eur. J. Bus. Soc. Sci. 2 101-19

Daniel J and Martin J H (2018). Hidden Markov Models Speech Lang. Process. 117

Dijkman R and Ipeirotis P (2015). Using Twitter to predict sales: a case study ar Xiv Prepr. arXiv 1503.04599. 471, 1-14

Fesenmaier D R, Pan B and Xiang Z (2016). Destination Competitiveness and Search Engine Marketing

Huang Y, B S Z, Huang K and Guan J (2005). Database Systems for Advanced Applications 3453 435-51

Jun S P, Yoo H S and Choi S (2018). Ten years of research change using Google Trends: From the perspective of big data utilizations and applications Technol. Forecast. Soc. Change, 130, 69-87

Khadivi P, Tech V and Tech V (2016). Wikipedia in the Tourism Industry: Forecasting Demand and Modeling Usage Behavior Proc. 30th Conf. Artif. Intell. (AAAI 2016) 4016-21

Length F (2016). Tourism forecasting by search engine data with noise-processing. $10114-30$

Li X, Pan B, Law R and Huang X (2017). Forecasting tourism demand with composite search index. Tour. Manag. 59 57-66

Mathew W, Raposo R and Martins B (2012). Predicting future locations with hidden Markov models UbiComp'12 - Proc. 2012 ACM Conf. Ubiquitous Comput. $911-8$

Nath B (2005). Stock Market Forecasting Using Hidden Markov Model : A New Approach

Nenonen S, Rasila H and Junnonen J M (2008). Customer Journey - a method to investigate user experience W111 Res. Rep. Usability Work. Phase, 2, 54-63 
Pai P F, Hong L C and Lin K P (2018). Using Internet Search Trends and Historical Trading Data for Predicting Stock Markets by the Least Squares Support Vector Regression Model Comput. Intell. Neurosci. 2018

Paper W (2016). Forecasting travelers in Spain with Google queries Máximo Camacho and Matías Pacce

Polykalas S E, Prezerakos G N and Konidaris A (2013). An algorithm based on Google Trends' data for future prediction. Case study: German elections IEEE Int. Symp. Signal Process. Inf. Technol. IEEE ISSPIT, 69-73

Rödel E (2017). Forecasting tourism demand in Amsterdam with Google Trends 170

SMITH S W (1946). The hypopietic patient. Practitioner, 156, 381-3

Song $\mathrm{H}$ and Li G (2008). Tourism demand modelling and forecasting-A review of recent research. Tour. Manag. 29 203-20

Song H (2010). Tourism Forecasting : Accuracy of Alternative Econometric Models Revisited Symp. A Q. J. Mod. Foreign Lit.

Time B T (2017). Fw: [ TELKOMNIKA ] \# 5993: Foreign Tourist Arrivals Forecasting Using Recurrent Neural Network Backpropagation Through Time To : Oger Vihikan

Volcheck E, Song H, Law R and Buhalis D (2018). Forecasting London Museum Visitors Using Google Trends Data e-Review. Tour. Res. 4

Wanjawa B W and Muchemi L (2014). ANN Model to Predict Stock Prices at Stock Exchange Markets 1-23

Wijnhoven F and Plant O (2017). Sentiment Analysis and Google Trends Data for Predicting. Car Sales ICIS. Proc. 1-16

Yang X, Pan B, Evans J A and Lv B (2015). Forecasting Chinese tourist volume with search engine data. Tour. Manag. 46, 386-97

Yang Y, Pan B and Song H (2014). Predicting Hotel Demand Using Destination Marketing Organization's Web Traffic Data J. Travel Res., 53, 433-47 